Journal Club

Editor's Note: These short, critical reviews of recent papers in the Journal, written exclusively by graduate students or postdoctoral fellows, are intended to summarize the important findings of the paper and provide additional insight and commentary. For more information on the format and purpose of the Journal Club, please see http://www.jneurosci.org/misc/ifa_features.shtml.

\title{
To What Extent Does Global Shape Influence Category Representation in the Brain?
}

\author{
N. Apurva Ratan Murty ${ }^{1}$ and R. T. Pramod ${ }^{2}$ \\ ${ }^{1}$ Graduate Program in Neuroscience, Centre for Neuroscience, and ${ }^{2}$ Graduate Program in Electrical and Communication Engineering, Department of \\ Electrical Communication Engineering, Indian Institute of Science, Bangalore, 560012, India \\ Review of Bracci and Op de Beeck
}

Humans are remarkably good at recognizing and rapidly categorizing objects into semantic categories (such as faces, cars, animals, etc), and this ability is thought to arise out of a series of increasingly complex hierarchical computations in the visual cortex. Visual information from the retina is relayed to the primary visual cortex after which it is further processed in two distinct visual processing streams: the dorsal "where" pathway and the ventral "what" pathway, predominantly involved in visual guidance of action and object recognition respectively. Neurons in the final stages of the ventral visual pathway-the inferior temporal cortex (ITC) in macaques and the lateral and ventral occipitotemporal cortex (LOC) in humans - respond to complex shapes, faces, and body parts. Although the visual features encoded in these higher regions remain unknown, it is believed that the representations also have a semantic organization that may

Received Feb. 4, 2016; revised March 4, 2016; accepted March 8, 2016.

N.A.R.M. is supported by a Senior Research Fellowship from the Council for Scientific and Industrial Research and the Ministry of Human Resource Development (MHRD) and R.T.P. is supported by a Junior Research Fellowship from the MHRD. We thank S. P. Arun and members of the laboratory (Zhivago, Harish, Melissa, Saurabh, and Sricharan) for comments and discussions.

The authors declare no competing financial interests.

Correspondence should be addressed to N. A. Ratan Murty, Centre for

Neuroscience, Indian Institute of Science, Bangalore, 560012, India. E-mail: ratan@cns.iisc.ernet.in.

DOI:10.1523/JNEUROSCI.0387-16.2016

Copyright $\odot 2016$ the authors $\quad 0270-6474 / 16 / 364149-03 \$ 15.00 / 0$ underlie our ability to rapidly categorize objects.

Evidence for a semantic representation in the ventral visual cortex, comes from macaque neurophysiology and functional magnetic resonance imaging (fMRI). When neurons in the ITC were probed with $\sim 1000$ naturalistic images, Kiani et al. (2007) observed that the responses to the animate and inanimate stimuli across the recorded ITC population were separable, indicating grouping for these categories. Within the animate category, they observed further subgrouping for faces, body parts, and hands. Comparing the responses of macaque ITC and human LOC using neuronal recordings and fMRI respectively, Kriegeskorte et al. (2008b) observed separable population activity patterns within ITC neurons and across LOC voxels for animate and inanimate objects, suggesting a similar category representation in human visual areas. These observations suggest that objects are organized along meaningful semantic categories in the brain. However, the simpler possibility is that objects within a category share common visual features (e.g., animals typically have a head with eyes, ears, nose, and mouth). Therefore, category grouping could arise because semantic and visual features are correlated.

In a recent study published in the Journal of Neuroscience, Bracci and Op de Beeck (2016) address this issue by uncoupling global shape and semantic categories, and characterizing shape and category representation in the dorsal and ventral visual pathways. The authors selected 54 stimuli: six categories (minerals, animals, fruits/vegetables, music, sporting equipment, and tools) and nine shapematched exemplars across categories. Subjects performed two behavioral tasks in which they were instructed to arrange stimuli on a computer monitor according to either shape or category similarity (Kriegeskorte and Mur, 2012). The pairwise distances between the stimuli at the end of the task were used to construct behavioral pairwise dissimilarity matrices for shape and category. The low-level shape similarity between the stimuli was also measured by calculating the pairwise difference in pixel intensities (silhouette shape representation). Very importantly, shape (silhouette shape and behavioral shape) and category dissimilarities were not correlated.

Brain activation in response to the stimuli was obtained using an eventrelated fMRI design, where subjects viewed the stimuli while performing a task indicating whether the presented image was smaller or larger than the previous image. The investigators identified 15 regions-of-interest (ROIs) in the lateral and ventral occipitotemporal cortex (ventral visual pathway) and parts of the frontal and parietal cortices (dorsal pathway) using either an 
independent functional localizer task or predefined anatomical masks. The blood oxygenation level-dependent response for each stimulus was extracted for every $3 \mathrm{D}$ volume, or voxel, in these ROIs. To obtain a pairwise neural dissimilarity matrix for each ROI, the response to one stimulus was correlated with the response to another stimulus across voxels within an ROI and subtracted from 1. This method facilitated direct comparisons between the computational (silhouette dissimilarity), behavioral (shape and category dissimilarity), and functional (ROI) dissimilarities (Kriegeskorte, 2008a). The authors reasoned that a significant positive correlation between the behavioral (shape/ category) and functional dissimilarity would indicate the selectivity of an ROI for shape and/or category information.

This analysis revealed a systematic organization of shape and category across the visual pathways. In the ventral visual pathway, the strength of shape encoding decreased and category encoding increased along the posterior-anterior anatomical axis. Along the dorsal pathway too, the posterior parietal areas encoded both shape and category, whereas the anterior frontal areas represented only category. However, a correlation between the behavioral and functional dissimilarities across ROIs does not imply that the category/shape information content is similar across ROIs. Addressing this aspect, the authors performed an unsupervised hierarchical clustering analysis on the functional dissimilarities obtained from the category and shape-selective ROIs separately. This analysis for the categoryselective ROIs revealed two clusters, the anterior-ventral ROIs and the frontal and parietal dorsal ROIs, indicating that the category information content was distinct across the dorsal and ventral ROIs. Following this, the authors identified that the anterior-ventral ROIs represented animate-inanimate category distinctions (e.g., animals vs sporting equipment), whereas the frontal and parietal dorsal ROIs represented action-nonaction category distinctions (e.g., tools vs minerals) more strongly than other category distinctions. Similarly, a hierarchical clustering analysis on the functional dissimilarities obtained from the shape selective ROIs revealed two clusters of ROIs, the posteriorventral and the anterior-ventral ROIs, indicating distinct shape representations in these regions: the posterior-ventral ROIs showed a greater match to a lowlevel silhouette shape dissimilarity and the anterior-ventral ROIs showed a greater match to the human behavioral shape dissimilarity, i.e., the shape dissimilarity as scored by human subjects. The authors were also able to assess the relation between category and high-level shape selectivity in each shape responsive ROI. Specifically, dissimilarity relationships due to mid- and high-level feature representations were obtained by subtracting the contribution of pixel-wise dissimilarities from behavioral shape dissimilarities (called the ps-index) and compared with functional dissimilarities in each ROI. Interestingly, this correlation increased along the visual shape selective hierarchy in a manner similar to that of category dissimilarities implying that higher-level shape properties are also represented in category selective regions.

Thus, the results can be summarized as follows: (1) category representations in the category-sensitive dorsal and ventral regions are different: the dorsal regions reveal stronger action-nonaction category distinctions, whereas ventral regions reveal stronger animate-inanimate category distinctions. (2) Shape representation along the ventral visual pathway changes from a low-level representation that matches the silhouette representation in the posterior areas, to a high-level representation that matches the perceptual shape representation in the anterior areas. (3) Both category and high-level shape properties explain neural representations in category selective ROIs.

These results, when considered in isolation, are not surprising. They agree well with previous studies that have observed similar distinctions between category representations in the dorsal and ventral visual areas and hierarchically increasing shape complexity along the ventral visual stream (Logothetis and Sheinberg, 1996; Patterson et al., 2007; Kriegeskorte et al., 2008b). The merit of the paper however, is that these results have been obtained after controlling for global shape across categories, a serious confound in earlier studies. Does this mean that the observed category selectivity cannot be explained by visual similarity between objects within the category? Although global shape might indeed be the strongest visual attribute, the results obtained do not exclude the possibility of other local attributes influencing the observed category distinctions. For example, minerals contain internal details with polygonal structures, animals have clear textural elements, such as fur, scales, or body patterns, and action-related items have handles and so on. The fact that shape and category representations have been observed in visual areas of the anterior ventral pathway suggests that the visual system could potentially be weighing in contributions from other visual attributes apart from global shape. Hence, to claim that category representation is independent of visual attributes will require systematic analysis of other attributes that correlate across categories. However, it may be impossible to disentangle all visual attributes from category representation as visual attributes themselves define these categories.

In the present study, the authors divide the whole brain into various ROIs and test the strength of category and shape representations in each ROI by correlating with behavioral measurements but this method has limitations. The correlation is sensitive to noise, which makes comparisons across ROIs difficult and the analysis ignores other non-ROI regions involved in shape and/or category representation. This potential concern however has been addressed in a recent study (Kaiser et al., 2016) that obtained brain-wide fMRI and magnetoencephalography (MEG) signals on a dataset which uncoupled shape from category by using visually similar categories (hands, gloves, shirts, and torsos). Specifically, the investigators used a searchlight approach to identify brain regions that represented shape and category related information independent of each other. The results, obtained using this unbiased approach, corroborate the results obtained by Bracci and Op de Beeck (2016): the posterior ventral visual regions encode shape and the anterior ventral regions encode category independently.

To summarize, this study (Bracci and Op de Beeck, 2016) is an important contribution to the field as it shows that category representations cannot be explained entirely by global shape. The study also raises many intriguing questions regarding category and object representations in the brain: do visual attributes give rise to a category representation or do categories influence how visual attributes are represented? Either way, what are the organizing principles of the higher visual areas that support such representations? This study has opened multiple exciting avenues about shape and category representations for further examination. 


\section{References}

Bracci S, Op de Beeck H (2016) Dissociations and associations between shape and category representations in the two visual pathways. J Neurosci 36:432-444. CrossRef Medline

Kaiser D, Azzalini DC, Peelen M V (2016) Shape-independent object category responses revealed by MEG and fMRI decoding. J Neurophysiol. In press. Retrieved January 6, 2016. CrossRef Medline

Kiani R, Esteky H, Mirpour K, Tanaka K (2007)

Object category structure in response patterns of neuronal population in monkey inferior temporal cortex. J Neurophysiol 97:42964309. CrossRef Medline

Kriegeskorte N, Mur M (2012) Inverse MDS: inferring dissimilarity structure from multiple item arrangements. Front Psychol 3:245. CrossRef Medline

Kriegeskorte N, Mur M, Bandettini P (2008a) Representational similarity analysis-connecting the branches of systems neuroscience. Front Syst Neurosci 2:4. CrossRef Medline

Kriegeskorte N, Mur M, Ruff DA, Kiani R, Bo- durka J, Esteky H, Tanaka K, Bandettini PA (2008b) Matching categorical object representations in inferior temporal cortex of man and monkey. Neuron 60:1126-1141. CrossRef Medline

Logothetis NK, Sheinberg DL (1996) Visual object recognition. Annu Rev Neurosci 19: 577-621. CrossRef Medline

Patterson K, Nestor PJ, Rogers TT (2007) Where do you know what you know? The representation of semantic knowledge in the human brain. Nat Rev Neurosci 8:976-987. CrossRef Medline 